\title{
SCREENING OF ANTIBACTERIAL ACTIVITY OF FUCOXANTHIN
}

\section{Tomasz M. Karpiński ${ }^{1 *}$, Artur Adamczak²}

${ }^{1}$ Department of Medical Microbiology, Poznań University of Medical Sciences, Wieniawskiego 3, 61-712 Poznań, Poland, e-mail: tkarpin@ump.edu.pl

${ }^{2}$ Department of Botany, Breeding and Agricultural Technology of Medicinal Plants, Institute of Natural Fibres and Medicinal Plants, Kolejowa 2, 62-064 Plewiska,

Poland, e-mail: artur.adamczak@iwnirz.pl

- INTRODUCTION: Fucoxanthin is an orange-coloured pigment present in brown algae (Phaeophyceae) and diatoms (Bacillariophyceae). This non-provitamin A carotenoid shows various biological activities, including antioxidant, anti-inflammatory, anti-obesity, anti-diabetic, and anticancer properties. Its antimicrobial effect is not sufficiently confirmed. Therefore, the purpose of the present work was to determine the activity of fucoxanthin against clinical strains of 20 species of bacteria.

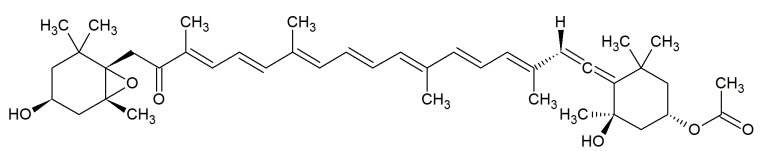

Chemical structure of fucoxanthin.

- METHODS: In the study, there were tested six Gram-positive bacteria (Enterococcus faecalis, Staphylococcus aureus, S. epidermidis, Streptococcus agalactiae, S. pneumoniae, S. pyogenes), and seven Gram-negative ones (Acinetobacter Iwoffii, Escherichia coli, Klebsiella oxytoca, K. pneumoniae, Proteus mirabilis, Pseudomonas aeruginosa, Serratia marcescens). Additionally, we investigated seven strict anaerobic pathogens (Actinomyces israelii, Atopobium parvulum, Mitsuokella multacida, Peptococcus niger, Porphyromonas gingivalis, Propionibacterium acnes, Veilonella parvula). The microbial growth inhibitory potential of fucoxanthin was determined according to recommendations of the Clinical and Laboratory Standards Institute (CLSI) and as described in our previous publication $[1,2]$ using the agar disc-diffusion and the micro-dilution methods.

- RESULTS: The obtained results indicated the biological activity of fucoxanthin against all tested strains of aerobic bacteria. The mean diameter of zone of inhibition (ZOI) ranged from 7.2 (P. mirabilis) to $12.2 \mathrm{~mm}$ (S. agalactiae), while the minimal inhibitory concentration (MIC) varied between 62.5 (S. agalactiae) and $500 \mu \mathrm{g} / \mathrm{mL}$ (P. mirabilis, P. aeruginosa, S. marcescens). Statistically significant stronger effect was observed in the case of Gram-positive (mean of ZOls $=10.5 \mathrm{~mm}$ ) than Gram-negative bacteria $(8.3 \mathrm{~mm})$. On the other hand, fucoxanthin was not active against strict anaerobic bacteria with the ZOIs of $6.0 \mathrm{~mm}$ and the MICs above $1000 \mu \mathrm{g} / \mathrm{mL}[3]$

Antibacterial activity of fucoxanthin determined by the agar disc-diffusion and micro-dilution methods.

\begin{tabular}{|c|c|c|}
\hline Studied bacterial strains & Zone of growth inhibition ZOI $(\mathbf{m m})$ & Minimal inhibitory concentration $\mathbf{M I C}(\mathbf{\mu g} / \mathbf{m L})$ \\
\hline Gram-positive & & $125-250$ \\
\hline Enterococcus faecalis & $9.0 \pm 0.89$ & 125 \\
\hline Staphylococcus aureus & $11.0 \pm 0.63$ & 125 \\
\hline Staphylococcus epidermidis & $11.2 \pm 0.75$ & 62.5 \\
\hline Streptococcus agalactiae & $12.2 \pm 0.75$ & 125 \\
\hline Streptococcus pneumoniae & $9.7 \pm 0.52$ & 125 \\
\hline Streptococcus pyogenes & $10.0 \pm 0.63$ & - \\
\hline Mean of all ZOls & $10.5 \pm 1.25$ & 125 \\
\hline Median & 10.0 & 250 \\
\hline Gram-negative & & 125 \\
\hline Acinetobacter /woffii & $8.2 \pm 0.41$ & $125-250$ \\
\hline Escherichia coli & $10.2 \pm 0.75$ & 250 \\
\hline Klebsiella oxytoca & $9.2 \pm 0.75$ & 500 \\
\hline Klebsiella pneumoniae & $8.8 \pm 0.75$ & $250-500$ \\
\hline Proteus mirabilis & $7.2 \pm 0.41$ & 500 \\
\hline Pseudomonas aeruginosa & $7.5 \pm 0.55$ & - \\
\hline Serratia marcescens & $7.3 \pm 0.52$ & 250 \\
\hline Mean of all ZOls & $8.3 \pm 1.18$ & $>1000$ \\
\hline Median & 8.0 & $>1000$ \\
\hline Anaerobic & & $>1000$ \\
\hline Actinomyces israelii & 6.0 & $>1000$ \\
\hline Atopobium parvulum & 6.0 & $>1000$ \\
\hline Mitsuokella multacida & 6.0 & $>1000$ \\
\hline Peptococcus niger & 6.0 & $>1000$ \\
\hline Porphyromonas gingivalis & 6.0 & \\
\hline Propionibacterium acnes & 6.0 & - \\
\hline Veilonella parvula & 6.0 & \\
\hline Negative control & $6.00 \pm 0.00$ & \\
\hline 20\% DMSO & & \\
\hline & & \\
\hline & &
\end{tabular}

- CONCLUSION: In conclusion, our study confirmed the antimicrobial activity of fucoxanthin. It seems that this substance can be a good antibacterial agent on some Gram-positive pathogens such as S. agalactiae, S. epidermidis, S. aureus, and weaker against Gram-negative bacteria.

[1] Karpiński T.M., Adamczak A. Antibacterial activity of ethanolic extracts of some moss species. Herba Pol. 2017, 63, 11-17. https://doi.org/10.1515/hepo-2017-0014 [2] Karpiński T.M. Efficacy of octenidine against Pseudomonas aeruginosa strains. Eur J Biol Res. 2019, 9, 135-140. http://dx.doi.org/10.5281/zenodo.3339499

[3] Karpiński T.M., Adamczak A. Fucoxanthin - an antibacterial carotenoid. Antioxidants 2019; 8(8): 239. https://doi.org/10.3390/antiox8080239 